Zbigniew Grande, Institute of Mathematics, Bydgoszcz Academy, Plac

Weyssenhoffa 11, 85-072 Bydgoszcz, Poland. e-mail:

grande@wsp. bydgoszcz.pl

\title{
ON DISCRETE LIMITS OF SEQUENCES OF DARBOUX BILATERALLY QUASICONTINUOUS FUNCTIONS
}

\begin{abstract}
In this article we show that a function $f$, such that the complement of the set of points at which $f$ has the Darboux property and is bilaterally quasicontinuous is nowhere dense, must be the discrete limit of a sequence of bilaterally quasicontinuous Darboux functions. Moreover, there is given a construction of a function that is the discrete limit of a sequence of bilaterally quasicontinuous Darboux functions and which does not have a local Darboux property on a dense set.
\end{abstract}

Let $\mathcal{R}$ be the set of all reals. In the article [3] the authors introduced the notion of the discrete convergence of sequences of functions and investigated the discrete limits in different families, for example in the family $\mathcal{C}$ of all continuous functions.

We will say that a sequence of functions $f_{n}: \mathbb{R} \rightarrow \mathbb{R}, n=1,2, \ldots$, discretely converges to the limit $f\left(f=d-\lim _{n \rightarrow \infty} f_{n}\right)$ if

$$
\forall_{x} \exists_{n(x)} \forall_{n>n(x)} f_{n}(x)=f(x) .
$$

For any family $\mathcal{P}$ denote by $B_{d}(\mathcal{P})$ the family of all discrete limits of sequences of functions from the family $\mathcal{P}$.

In [3] the class $B_{d}(\mathcal{C})$ is described and the authors observe that every strictly increasing function $f$ whose the set of discontinuity points is dense does not belong to the discrete Baire system generated by $\mathcal{C}$ and the discrete convergence.

A function $f: \mathbb{R} \rightarrow \mathbb{R}$ is quasicontinuous (bilaterally quasicontinuous) at a point $x$ if for every positive real $\eta$ there is a nonempty open set $U \subset(x-\eta, x+\eta)$

Key Words: Discrete convergence, quasicontinuity, bilateral quasicontinuity, Darboux property.

Mathematical Reviews subject classification: 26A15, 26A21, 26A99.

Received by the editors September 8, 2000

* Supported by Bydgoszcz Academy grant 2000 
(there are nonempty open sets $V \subset(x-\eta, x)$ and $W \subset(x, x+\eta))$ such that $f(U) \subset(f(x)-\eta, f(x)+\eta)(f(V \cup W) \subset(f(x)-\eta, f(x)+\eta))([5,6])$.

In [4] it is proved that

(1) A function $f: \mathbb{R} \rightarrow \mathbb{R}$ is the discrete limit of a sequence of quasicontinuous functions if and only if the set

$$
D_{q}(f)=\{x ; f \text { is not quasicontinuous at } x\}
$$

is nowhere dense.

(2) A function $f: \mathbb{R} \rightarrow \mathbb{R}$ is the discrete limit of a sequence of bilaterally quasicontinuous functions if and only if the set

$$
D_{b q}(f)=\{x ; f \text { is not bilaterally quasicontinuous at } x\}
$$

is nowhere dense.

Let $\mathcal{D}$ denote the class of all functions $f: \mathbb{R} \rightarrow \mathbb{R}$ having Darboux property and let $Q$ (respectively $Q_{b}$ ) be the family of all quasicontinuous (bilaterally quasicontinuous) functions.

In [7] the author investigates some classes $\mathcal{P}$ of functions from $\mathbb{R}$ to $\mathbb{R}$ such that $\mathcal{P} \subset B_{d}(\mathcal{D} \cap \mathcal{P})$. But neither of the classes $Q$ and $Q_{b}$ satisfies the hypothesis of that general theorem from [5]. For this observe that $Q \cap \mathcal{D} \subset Q_{b}$ and that, for each continuous from the right hand and increasing function $f: \mathbb{R} \rightarrow \mathbb{R}$ discontinuous on a dense set, we have

$$
D_{q}(f)=\emptyset \text { and the set } D_{b q}(f) \text { is dense. }
$$

Consequently,

$$
Q \backslash B_{d}(\mathcal{D} \cap Q)=Q \backslash B_{d}\left(\mathcal{D} \cap Q_{b}\right) \neq \emptyset .
$$

In this article I show two theorems describing the class $B_{d}\left(\mathcal{D} \cap Q_{b}\right)$. In our considerations we will apply the following notations: Let $f: \mathbb{R} \rightarrow \mathbb{R}$ be a function and let $x \in \mathbb{R}$ be a point. Put

$$
\begin{aligned}
& K^{+}(f, x)=\left\{y: \exists_{\left(x_{n}\right)} x<x_{n} \rightarrow x \text { and } y=\lim _{n \rightarrow \infty} f\left(x_{n}\right)\right\}, \\
& K^{-}(f, x)=\left\{y: \exists_{\left(x_{n}\right)} x>x_{n} \rightarrow x \text { and } y=\lim _{n \rightarrow \infty} f\left(x_{n}\right)\right\},
\end{aligned}
$$

and recall that $x$ is a Darboux point of a function $f$ if for every positive real $r$ and for all reals $a \in\left(\min \left(f(x), \inf \left(K^{+}(f, x)\right)\right), \max \left(f(x), \sup \left(K^{+}(f, x)\right)\right)\right)$ and $b \in\left(\min \left(f(x), \inf \left(K^{-}(f, x)\right)\right), \max \left(f(x), \sup \left(K^{-}(f, x)\right)\right)\right)$ there are points $c \in(x, x+r)$ and $d \in(x-r, x)$ such that $f(c)=a$ and $f(d)=b$. It is known 
$([2,1])$ that a function $f$ has the Darboux property if and only if each point $x$ is a Darboux point of a function $f$.

Let

$$
\operatorname{Dar}(f)=\{x: x \text { is not a Darboux point of } f\} .
$$

Theorem 1. Let a function $f: \mathbb{R} \rightarrow \mathbb{R}$ be such that the set $\operatorname{Dar}(f) \cup D_{b q}$ is nowhere dense. Then $f$ is the discrete limit of a sequence of Darboux bilaterally quasicontinuous functions.

Proof. Denote by $A$ the closure $\operatorname{cl}\left(\operatorname{Dar}(f) \cup D_{b q}\right)$ of the union $\operatorname{Dar}(f) \cup D_{b q}$. Let $\left(I_{n}\right)$ be a sequence of all components of the set $\mathbb{R} \backslash A$. If $I_{n}=\left(a_{n}, b_{n}\right)$ and $a_{n}, b_{n} \in \mathbb{R}$ then we find two sequences of closed intervals $J_{n, k}=\left[c_{n, k}, d_{n, k}\right]$ and $L_{n, k}=\left[p_{n, k}, q_{n, k}\right], k=1,2, \ldots$, such that

$$
\begin{aligned}
& a_{n}<d_{n, k+1}<c_{n, k}<d_{n, k} \text { and } d_{n, 1}<p_{n, k}<q_{n, k}<p_{n, k+1}<b_{n} \text { for } \\
& k=1,2, \ldots ; \\
& a_{n}=\lim _{k \rightarrow \infty} d_{n, k} \text { and } b_{n}=\lim _{k \rightarrow \infty} p_{n, k} ; \\
& f \text { is continuous at all points } c_{n, k}, d_{n, k}, p_{n, k}, q_{n, k}, k, n \geq 1 .
\end{aligned}
$$

If $a_{n}=-\infty$ (or $\left.b_{n}=\infty\right)$ then we find only one sequence $\left(L_{n, k}\right)$ (or respectively $\left.\left(J_{n, k}\right)\right)$.

For all $n, k$ define continuous functions $f_{n, k}: J_{n, k} \rightarrow \mathbb{R}$ and $g_{n, k}: L_{n, k} \rightarrow \mathbb{R}$ such that

$$
f_{n, k}\left(J_{n, k}\right)=g_{n, k}\left(L_{n, k}\right) \supset[-k, k]
$$

and

$$
\begin{gathered}
f_{n, k}\left(c_{n, k}\right)=f\left(c_{n, k}\right), \quad f_{n, k}\left(d_{n, k}\right)=f\left(d_{n, k}\right), \\
g_{n, k}\left(p_{n, k}\right)=f\left(p_{n, k}\right) \text { and } g_{n, k}\left(q_{n, k}\right)=f\left(q_{n, k}\right) .
\end{gathered}
$$

For $m=1,2, \ldots$ let

$$
f_{m}(x)= \begin{cases}f_{n, k}(x) & \text { for } x \in J_{n, k}, \text { where } n \geq 1 \text { and } \geq m \\ g_{n, k}(x) & \text { for } x \in L_{n, k}, \text { where } n \geq 1 \text { and } \geq m \\ f(x) & \text { otherwise on } \mathbb{R} .\end{cases}
$$

Since for each $m \geq 1$, we have $A \supset \operatorname{Dar}\left(f_{m}\right) \cup D_{b q}\left(f_{m}\right)$ and

$$
K^{+}\left(f_{m}, x\right) \cap K^{-}\left(f_{m}, x\right)=[-\infty, \infty] \text { for each } x \in A,
$$

every function $f_{m} \in \mathcal{D} \cap Q_{b}$.

Evidently, $f$ is the discrete limit of the sequence $\left(f_{m}\right)$. 
Theorem 2. There is a function $f: \mathbb{R} \rightarrow \mathbb{R}$ belonging to $B_{d}\left(\mathcal{D} \cap Q_{b}\right)$ such that the set $\operatorname{Dar}(f)$ is dense.

Proof. Let $\left(I_{n}\right)$ be an enumeration of all open intervals with rational endpoints. In the first step we find a nonempty perfect nowhere dense set $C_{1} \subset I_{1}$ and a nowhere constant function $f_{1}: \mathbb{R} \rightarrow[-1,1] \backslash\{0\}$ such that:

the restricted function $f_{1} /\left(\mathbb{R} \backslash C_{1}\right)$ is continuous;

$f_{1}(\mathbb{R})=[-1,0) \cup(0,1] ;$

for every $x \in C_{1}$ being a bilateral accumulation point of $C_{1}$ and for all reals $r>0$ and $y \in(-1,0) \cup(0,1)$ there are points $a \in(x-r, x)$ and $b \in(x, x+r)$ such that $f_{1}(a)=y=f_{1}(b)$;

if $x \in C_{1}$ is unilaterally isolated in $C_{1}$ then $f_{1}(x)$ is an irrational number.

Next we fix a positive integer $n>1$ and we suppose that for all positive integers $1<k<n$ we have defined closed intervals

$$
J_{k} \subset I_{k} \backslash \bigcup_{i<k} C_{i}
$$

nonempty perfect nowhere dense sets $C_{k} \subset \operatorname{Int}\left(J_{k}\right)$, where $\operatorname{Int}\left(J_{k}\right)$ denotes the interior of the interval $J_{k}$, closed intervals $M_{k}$, rationals $w_{k} \in \operatorname{Int}\left(M_{k}\right)$ and nowhere constant functions $f_{k}: \mathbb{R} \rightarrow[-1,1]$ such that:

the length $d\left(J_{k}\right)$ of the interval $J_{k}$ is less then $\frac{1}{k}$;

for $k<n-1$ we have $f_{k}(x)=f_{k+1}(x)$ for $x \in \mathbb{R} \backslash \operatorname{Int}\left(J_{k+1}\right)$;

$f_{k}\left(J_{k}\right)=M_{k} \backslash\left\{w_{k}\right\}$, where $w_{0}=0 ;$

$o s c_{J_{k+1}} f_{k}<\frac{2}{8^{k}} \min \left\{\operatorname{dist}\left(w_{i}, f_{k}\left(J_{k+1}\right)\right)=\inf \left\{\left|w_{i}-f_{k}(x)\right| ; x \in J_{k+1}\right\} ; i \leq\right.$ $k\}$ for $k<n-1$;

$M_{1}=[-1,1]$ and for $k>1$ the set $M_{k} \subset[-1,1] \backslash\left\{w_{i} ; i<k\right\}$ is the interval containing $f_{k-1}\left(J_{k}\right)$ of the length less than

$$
\frac{2}{8^{k-1}} \min \left(\left\{\operatorname{dist}\left(w_{i}, M_{k}\right)=\inf \left\{\left|x-w_{i}\right| ; x \in M_{k}\right\} ; i<k\right\}\right)
$$

with the same center as the center of the interval $f_{k-1}\left(J_{k}\right)$;

the functions $f_{k}$ are continuous at all points $x \in \mathbb{R} \backslash \bigcup_{i \leq k} C_{i}$; 
for $i \leq k<n$, for each point $x \in C_{i}$ being a bilateral accumulation point of $C_{i}$, for each positive real $r$ and for every point $y \in \operatorname{Int}\left(M_{i}\right) \backslash\left\{w_{i}\right\}$ there are points $a_{i} \in(x-r, x)$ and $b_{i} \in(x, x+r)$ such that $f_{k}\left(a_{i}\right)=f_{k}\left(b_{i}\right)=y$;

if a point $x \in C_{k}$ is isolated from the right (from the left) hand in $C_{k}$, $k<n$, then $f_{k}(x)$ is irrational and for each positive real $r<\operatorname{dist}\left(x, \mathbb{R} \backslash J_{k}\right)$ the interval $(x, x+r) \subset \operatorname{Int}\left(J_{k}\right) \backslash C_{k}$ and the image

$$
\begin{aligned}
& f_{k}([x, x+r)) \subset\left(\min \left(M_{k}\right), w_{k}\right) \text { or } f_{k}([x, x+r)) \subset\left(w_{k}, \max \left(M_{k}\right)\right) \\
& \left((x-r, x) \subset \operatorname{Int}\left(J_{k}\right) \backslash C_{k}\right. \text { and } \\
& \quad f_{k}((x-r, x]) \subset\left(\min \left(M_{k}\right), w_{k}\right) \text { or } f_{k}((x-r, x]) \subset\left(w_{k}, \max \left(M_{k}\right)\right) .
\end{aligned}
$$

Now, in the step $n$ we find a closed interval $J_{n}$ such that

$$
J_{n} \subset I_{n} \backslash \bigcup_{k<n} C_{k}, \text { and } d\left(J_{n}\right)<\frac{1}{n},
$$

and

$$
\operatorname{osc}_{J_{n}} f_{n-1}<s_{n}=\frac{2}{8^{n-1}} \min \left\{\operatorname{dist}\left(w_{i}, f_{n-1}\left(J_{n}\right)\right) ; i<n\right\} .
$$

Let $M_{n} \subset[-1,1] \backslash\left\{w_{i} ; i<n\right\}$ be a closed interval of the length $d\left(M_{n}\right)$ such that $d\left(f_{n-1}\left(J_{n}\right)\right)<d\left(M_{n}\right)<s_{n}$ with the same center as the interval $f_{n-1}\left(J_{n}\right)$ and let $C_{n} \subset \operatorname{Int}\left(J_{n}\right)$ be a nonempty nowhere dense perfect set. Fix a rational point $w_{n} \in \operatorname{Int}\left(M_{n}\right)$ and let

$$
\min \left(M_{n}\right)=v_{0}<v_{1}=w_{n}<v_{2}=\max \left(M_{n}\right) .
$$

The family $\left\{T_{k}\right\}_{k}$ of all components of the set $\operatorname{Int}\left(J_{n}\right) \backslash C_{n}$ is the union of pairwise disjoint subfamilies $\left\{T_{i, j}\right\}_{j}, i \leq 2$, such that

$$
\forall_{i \leq 2} C_{n} \subset \operatorname{cl}\left(\bigcup_{j} T_{i, j}\right)
$$

where $\operatorname{cl}(X)$ denotes the closure of the set $X$.

For $i \leq 2$ and $j=1,2, \ldots$ we define nowhere constant continuous functions $f_{n, i, j}: T_{i, j} \rightarrow\left(v_{i-1}, v_{i}\right)$ such that

$f_{n, i, j}\left(T_{i, j}\right)=\left(v_{i-1}, v_{i}\right)$ for all $i \leq 2$ and $j \geq 1$;

if $T_{i, j}=\left(a_{i, j}, b_{i, j}\right)$ and $a_{i, j}$ (or resp. $\left.b_{i, j}\right)$ is an endpoint of the interval $J_{n}$ then

$$
\lim _{x \rightarrow a_{i, j}+} f_{n, i, j}(x)=f_{n-1}\left(a_{i, j}\right)
$$


(or resp.

$$
\left.\lim _{x \rightarrow b_{i, j}-} f_{n, i, j}(x)=f_{n-1}\left(b_{i, j}\right)\right) ;
$$

if $T_{i, j}=\left(a_{i, j}, b_{i, j}\right)$ and $a_{i, j} \in C_{n}$ (or resp. $\left.b_{i, j} \in C_{n}\right)$ then for every $y \in$ $\left(v_{i-1}, v_{i}\right)$ and for each positive real $r$ there is a point $c \in\left(a_{i, j}, \min \left(a_{i, j}+\right.\right.$ $\left.\left.r, b_{i, j}\right)\right)$ (or resp. $\left.d \in\left(\max \left(a_{i, j}, b_{i, j}-r\right), b_{i, j}\right)\right)$ such that $f_{n, i, j}(c)=y$ (or resp. $\left.y=f_{n, i, j}(d)\right)$.

Let $f_{n}: \mathbb{R} \rightarrow[-1,1]$ be a function such that

$f_{n}$ is equal $f_{n-1}$ on the set $\mathbb{R} \backslash \operatorname{Int}\left(J_{n}\right)$ and is equal $f_{n, i, j}$ on the intervals $T_{i, j}, i \leq 2, j \geq 1$

if $T_{i, j}=\left(a_{i, j}, b_{i, j}\right)$ and $a_{i, j} \in C_{n}$ (or resp. $\left.b_{i, j} \in C_{n}\right)$ then $f_{n}\left(a_{i, j}\right) \in$ $\left(v_{i-1}, v_{i}\right)$ (or resp. $\left.f_{n}\left(b_{i, j}\right) \in\left(v_{i-1}, v_{i}\right)\right)$ is irrational;

$$
f_{n}\left(C_{n}\right)=M_{n} \backslash\left\{w_{n}\right\} .
$$

Finally we define $f=\lim _{n \rightarrow \infty} f_{n}$. Since

$$
\left|f_{n+1}-f_{n}\right| \leq s_{n}<\frac{2}{8^{n-1}} \text { for } n \geq 1,
$$

the sequence $\left(f_{n}\right)$ uniformly converges to $f$. From the construction follows that the functions $f_{n}, n=1,2, \ldots$, are bilaterally quasicontinuous. So $f$ is also a bilaterally quasicontinuous function. Since the images $f\left(J_{n}\right)$ of all intervals $J_{n} \subset I_{n}$ are not intervals $\left(w_{n}\right.$ is not in $f\left(J_{n}\right)$ ), the set $\operatorname{Dar}(f)$ is dense.

We will prove that $f \in B_{d}\left(\mathcal{D} \cap Q_{b}\right)$. For this observe that every set

$$
E_{n}=\left\{x \in C_{n} ; x \text { is a bilateral accumulation point of } C_{n}\right\},
$$

$n=1,2, \ldots$, is the union of pairwise disjoint sets $E_{n, k}, k=1,2, \ldots$, which are c-dense in $C_{n}$, i.e. for each open interval $I$ with $I \cap C_{n} \neq \emptyset$ and for each $k \geq 1$ the cardinality of the intersection $E_{n, k} \cap I$ is equal continuum.

For $n, k \geq 1$ there are functions $g_{n, k}: E_{n, k} \rightarrow M_{n}$ such that for each interval $I$ with $I \cap E_{n} \neq \emptyset$ the equality $g_{n, k}\left(I \cap E_{n, k}\right)=M_{n}$ is true. For $k \geq 1$ let

$$
g_{k}(x)= \begin{cases}g_{n, i}(x) & \text { for } x \in E_{n, i}, \text { where } i \geq k \text { and } n=1,2, \ldots \\ f(x) & \text { otherwise on } \mathbb{R} .\end{cases}
$$

Evidently, $f=d-\lim _{k \rightarrow \infty} g_{k}$. From the construction of $f$ follows that every function $g_{k}, k=1,2, \ldots$, is bilaterally quasicontinuous. We will prove that they have also the Darboux property. For this fix a positive integer $k$ and 
observe that the functions $f$ and consequently $g_{k}$ are continuous at all points $x \in \mathbb{R} \backslash \bigcup_{n} C_{n}$. So,

$$
\operatorname{Dar}(f) \subset \bigcup_{n} C_{n}
$$

If $x \in E_{n}$ for some positive integer $n$ then from the construction of the function $g_{k}$ and the properties of the functions $g_{n, k}$ follows that $x$ is not in $\operatorname{Dar}\left(g_{k}\right)$. So, we suppose that there is a positive integer $n$ such that $x$ belong to the difference $C_{n} \backslash E_{n}$. Then $x$ is isolated in $C_{n}$ from the right or from the left hand. If

Suppose that $x$ is isolated in $C_{n}$ from the left hand. Fix a positive real $r$.

$$
y \in \operatorname{Int}\left(K^{+}\left(g_{k}, x\right)\right) \ni g_{k}(x)
$$

then, by the construction of $g_{k}$ on $E_{n}$, follows that there is a decreasing sequence of points $x_{j} \in E_{n} \cap(x, x+r)$ such that

$$
\lim _{j \rightarrow \infty} x_{j}=x \text { and } g_{k}\left(x_{j}\right)=y \text { for } j \geq 1 .
$$

Moreover,

$$
g_{k}(x)=f(x)=f_{n}(x) \in \operatorname{Int}\left(M_{n}\right) \backslash\left\{w_{i} ; i \geq 1\right\},
$$

so for any

$$
y \in \operatorname{Int}\left(K^{-}\left(g_{k}, x\right)\right)=\operatorname{Int}\left(K^{-}(f, x)\right)=\operatorname{Int}\left(K^{-}\left(f_{n}, x\right)\right) \ni g_{k}(x)
$$

there is an increasing sequence of points

$$
t_{j} \in(x-r, x) \cap\left(J_{n} \backslash C_{n}\right)
$$

such that

$$
\lim _{j \rightarrow \infty} t_{j}=x \text { and } f_{n}\left(t_{j}\right)=y \text { for } j \geq 1 .
$$

If there is a positive integer $j$ with

$$
y=f_{n}\left(t_{j}\right)=f\left(t_{j}\right)=g_{k}\left(t_{j}\right)
$$

then in the considered case the proof is completed.

If not, there are positive integers $i>n$ and $j$ such that

$$
J_{i} \subset(x-r, x) \cap J_{n} \text { and } t_{j} \in \operatorname{Int}\left(J_{i}\right) .
$$

Consequently,

$$
y=f_{n}\left(t_{j}\right) \in \operatorname{Int}\left(M_{i}\right) \subset f_{i}\left(C_{i}\right)=f\left(C_{i}\right) \subset g_{k}\left(C_{i}\right),
$$


and there is a point

$$
z \in(x-r, x) \text { with } g_{k}(z)=y .
$$

So, $x$ is not in $\operatorname{Dar}\left(g_{k}\right)$.

The proof that any point $x \in C_{n}$ which is isolated in $C_{n}$ from the right hand belongs to $\mathbb{R} \backslash \operatorname{Dar}\left(g_{k}\right)$ is analogous. So, $\operatorname{Dar}\left(g_{k}\right)=\emptyset$ for all $k=1,2, \ldots$ and the proof is completed.

\section{References}

[1] Bruckner A.M.; Differentiation of real functions, Lectures Notes in Math. 659, Springer-Verlag, Berlin 1978.

[2] Bruckner A.M. and Ceder J.; Darboux continuity, Jber. Deut. Math. Ver. 67 (1965), 93-117.

[3] Császár A. and Laczkovich M.; Discrete and equal convergence, Studia Sci. Math. Hungar. 10 (1975), 463-472.

[4] Grande Z.; On discrete limits of sequences of approximately continuous and $T_{a e}$-continuous functions, to appear.

[5] Kempisty S.; Sur les fonctions quasi-continues, Fund. Math. 19 (1932), 184-197.

[6] Neubrunn T.; Quasi-continuity, Real Anal. Exch. 14 No.2 (1988-89), 259306.

[7] Strońska E.; Some remarks on discrete and uniform convergence, to appear. 